\title{
ROBUST CONTROL OF UNCERTAIN TIME-DELAY SYSTEMS VIA SLIDING MODE CONTROL AND LMI- Ho TECHNIQUE
}

Ming-Chang Pai

Department of Automation Engineering, Nan Kai Institute of Technology, Tsao-Tun, Taiwan, R.O.C., pmc@nkc.edu.tw

Follow this and additional works at: https://jmstt.ntou.edu.tw/journal

Part of the Electrical and Computer Engineering Commons

\section{Recommended Citation}

Pai, Ming-Chang (2008) "ROBUST CONTROL OF UNCERTAIN TIME-DELAY SYSTEMS VIA SLIDING MODE CONTROL AND LMI- Ho TECHNIQUE," Journal of Marine Science and Technology: Vol. 16: Iss. 1, Article 1.

DOI: 10.51400/2709-6998.1991

Available at: https://jmstt.ntou.edu.tw/journal/vol16/iss1/1

This Research Article is brought to you for free and open access by Journal of Marine Science and Technology. It has been accepted for inclusion in Journal of Marine Science and Technology by an authorized editor of Journal of Marine Science and Technology. 


\section{ROBUST CONTROL OF UNCERTAIN TIME-DELAY SYSTEMS VIA SLIDING MODE CONTROL AND LMI- $H_{\infty}$ TECHNIQUE}

\begin{abstract}
Key words: sliding mode control, state delays, mismatched parameter uncertainties, linear matrix inequality (LMI).

ABSTRACT

This paper presents a proportional-integral sliding mode control (PISMC) methodology for a class of linear uncertain systems with state delays. The systems are assumed to have structured, mismatched, and time-varying parameter uncertainties as well as mismatched and bounded external disturbances. Based on Lyapunov stability theorem and linear matrix inequality (LMI) $H_{\infty}$ technique, a sufficient condition is derived to guarantee the global stability of the dynamics and a prescribed $H_{\infty}$ norm bound of disturbance attenuation for all admissible uncertainties. Furthermore, this scheme assures robustness against state delays, parameter uncertainties and disturbances. Finally, an example is given to illustrate the feasibility of the proposed control methodology.
\end{abstract}

Ming-Chang Pai*

\section{INTRODUCTION}

Time delay can be found in various engineering systems such as the turbojet engine, rolling mill, chemical processes, manual control, and long transmission line in pneumatic, hydraulic systems [8]. The existence of time delay in a system frequently becomes a source of instability and poor performance. Furthermore, the stabilization of control systems which have delays is not as easy as that of systems without delays. Therefore, the control problem of time-delay systems has received considerable attention in recent years.

Sliding mode control (SMC) has mostly focused on systems without time delays. However time delays are common in practical application. Hence, some studies have used SMC to stabilize time-delay systems. Chou and Cheng [2] have developed an adaptive variable structure controller for perturbed time-varying state delay systems. Gouaisbaut et al. [4] devel-

Paper submitted 08/02/06; accepted 11/24/06. Author for correspondence: Ming-Chang Pai (e-mail: pmc@nkc.edu.tw).

*Department of Automation Engineering, Nan Kai Institute of Technology, Tsao-Tun, Taiwan, R.O.C. oped a robust control of delay systems by using a SMC design and LMI technique. Roh and Oh [10] developed a SMC for the robust stabilization of uncertain linear input-delay systems with nonlinear parametric perturbations. Xia et al. [15] have developed a SMC for linear systems with input and state delays. Al-Shamali et al. [1] have developed a SMC scheme to ensure the asymptotic stability of a linear system with delays in both the input and state variable. However, all of these papers are assumed the uncertainties are matched or do not exist.

A feature of SMC is that the closed-loop system can be designed to be robust with respect to parametric uncertainties and external disturbances, provided that their bounds are known and the matching condition [3] is satisfied. However, if the matching condition is not satisfied or the system suffers from mismatched uncertainties, then the system behavior will be affected by uncertainties. Shyu et al. [12] have developed a SMC for a system with mismatched uncertainties and the chattering phenomenon. Sam et al. [11] have developed a PISMC for a system with mismatched uncertainties. However, they did not deal with state delays. Li and DeCarlo [6,7] developed a robust SMC design method for a class of uncertain time-delay systems with multiple state delays. However, in their method, there is no synthesis, but only analysis. The disadvantageous oscillations (chattering) exist. Furthermore, they assumed external disturbances are matched.

In this paper, an approach is proposed to address the problem of stabilizing systems with state delays, mismatched uncertainties and disturbances. Based on Lyapunov stability theorem and LMI- $H_{\infty}$ technique $[5,16,17]$, the asymptotic stability of uncertain time-delay systems is guaranteed and a prescribed $H_{\infty}$ norm bound of disturbance attenuation is achieved. The proposed method has the following attractive features: 1) the order of the motion equation in the sliding mode is equal to the order of the original system, rather than reduced by the number of dimension of the control input. 2) the robustness of systems can be guaranteed throughout the entire response of systems starting from the initial time instance. 3) the control design is rather straightforward and the asymptotic stability of time-delay systems is guaranteed without state transformation. 4) it can be easily extended to the case of multiple state delays and suitable for the case of both mismatched and matched external distur- 
bances.

The remainder of the paper is organized as follows. Section 2 briefly states system description and problem formulation. Section 3 provides the switching surface design and stability analysis of the dynamics in the specified switching surface for all admissible uncertainties. Section 4 addresses the control law and the existence of sliding mode. Section 5 presents results from numerical simulations. Finally, a conclusion is provided in section 6.

\section{SYSTEM DESCRIPTION AND PROBLEM FOR- MULATION}

Consider an uncertain linear time-delay system described by the state equation

$$
\begin{gathered}
\dot{x}(t)=(A+\Delta A(t)) x(t)+\left(A_{d}+\Delta A_{d}(t)\right) x(t-d) \\
+(B+\Delta B(t)) u(t)+B_{1} w(t) \\
z(t)=C x(t) \\
x(t)=\varphi(t), t \in[-d, 0] .
\end{gathered}
$$

where $x(t) \in R^{n}$ is the state, $u(t) \in R^{m}$ is the control input, $w(t) \in R^{l}$ is the external disturbance, which belongs to $L_{2}[0, \infty)$, and $z(t) \in R^{p}$ is the controlled signal output. $A$, $A_{d}, B, B_{1}$ and $C$ are known constant matrices with appropriate dimensions, $d \geq 0$ is a known constant time delay, and $\varphi(t)$ is a continuous vector-valued initial state function. The terms $\Delta A(t), \Delta A_{d}(t)$ and $\Delta B(t)$ are unknown real-valued functions representing time-varying parameter uncertainties of the matrices $A, A_{d}$ and $B$, respectively.

For completing the description of uncertain dynamic system (1), the following assumptions are assumed to be valid:

Assumption 1. All of system states are measurable.

Assumption 2. The input uncertainty $\Delta B(t)$ is matched, i.e., there exists a matrix $N(t) \in R^{m \times m}$ such that $\Delta B(t)=B N(t)$ with $\|N(t)\| \leq \delta_{B}<1$ and $B+\Delta B(t)$ are assume to be full column rank for all $t$.

Assumption 3. (Structured perturbation) The time-varying parameter uncertainties $\Delta A(t)$ and $\Delta A_{d}(t)$ are assumed to be norm-bounded and with the form

$$
\Delta A(t)=D_{a} F_{a}(t) E_{a}, \Delta A_{d}(t)=D_{d} F_{d}(t) E_{d}
$$

where $D_{a}, D_{d}, E_{a}$ and $E_{d}$ are known constant matrices with appropriate dimensions, $F_{a}(t)$ and $F_{d}(t)$ are unknown matrix functions of uncertain parameters satisfying

$$
F_{a}^{T}(t) F_{a}(t) \leq I, F_{d}^{T}(t) F_{d}(t) \leq I
$$

where $I$ is an identify matrix with appropriate dimension.

It is assumed that all the elements of $F_{a}(t)$ and $F_{d}(t)$ are Lebesgue measurable. The uncertainties $\Delta A(t)$ and $\Delta A_{d}(t)$ are said to be admissible if both (2) and (3) are hold.

The objective of this paper is to develop a PI sliding mode controller which is robust to time-varying uncertainties and guarantees the closed-loop system is asymptotically stable as well as achieves, under zero initial conditions, a prescribed $H_{\infty}$ norm bound $\gamma$ of disturbance attenuation, i.e., $\|z(t)\|_{2}<\gamma\|w(t)\|_{2}$ for all nonzero $w(t) \in L_{2}[0, \infty)$.

\section{Definition}

Subject to the zero initial conditions, $x(t)=\varphi(t)=0$, $t \in[-d, 0]$, the controlled output $z(t)$ satisfies $\|z(t)\|_{2}<\gamma\|w(t)\|_{2}$ for any nonzero disturbance input $w(t) \in L_{2}[0, \infty)$ is said to be $\left\|T_{z w}\right\| \leq \gamma, \gamma>0$.

\section{SWITCHING SURFACE DESIGN}

In general, the designing procedure of SMC technique can be divided into two steps. The first step is to design a switching surface such that system response in the sliding mode has the desired properties, for example, stability, robustness, tracking capability, etc. The second step is to design the control law to globally drive the system trajectories to the specified sliding surface, and maintain there for all subsequent time.

In this paper, the PI switching function is defined as

$$
s(t)=G x(t)-G \int_{0}^{t}(A+B K) x(\tau) d \tau
$$

where $G \in R^{m \times n}$ is chosen such that the matrix $G B$ is nonsingular and $K \in R^{m \times n}$ is designed later such that system (1) in the sliding mode is asymptotically stable.

To facilitate further development, the following lemma is needed.

\section{Lemma 1 [9]}

For any constant $\varepsilon>0$ and real matrices $D, F(t)$, and $E$ of appropriate dimensions, suppose $F^{T}(t) F(t) \leq I_{n}$. Then

$$
D F(t) E+E^{T} F^{T}(t) D^{T} \leq \varepsilon D D^{T}+\frac{1}{\varepsilon} E^{T} E
$$

In the sequel, the conditions are derived to evaluate the stability and robustness of uncertain time-delay system (1) restricted to the switching surface $s(t)=0$ with the existence of appropriate positive definite matrices.

\section{Theorem 1}

Consider uncertain time-delay system (1) with $w(t)=0$, assumptions 1-3 and the PI switching function (4). Let $R \in R^{m \times m}$ and $R_{1} \in R^{n \times n}$ be given positive definite symmetric matrices. If there exist a positive constant $\varepsilon>0$, a positive-definite symmetric solution $P \in R^{n \times n}$ for any admissible 
uncertainty $F_{a}(\cdot), F_{d}(\cdot)$ such that the following inequality is satisfied

$$
\Theta=A^{T} P+P A-2 P B R^{-1} B^{T} P+P L_{1} P+Q_{1}<0
$$

where

$$
\begin{gathered}
L_{1}=\varepsilon\left(\tilde{D}_{a} \tilde{D}_{a}^{T}+\tilde{A}_{d} \tilde{A}_{d}^{T}+\tilde{D}_{d} \tilde{D}_{d}^{T}\right) \\
Q_{1}=\frac{1}{\varepsilon}\left(I+E_{a}^{T} E_{a}+E_{d}^{T} E_{d}\right) \\
\tilde{A}_{d}=\left[I-B(G B)^{-1} G\right] A_{d} \\
\tilde{B}_{1}=\left[I-B(G B)^{-1} G\right] B_{1} \\
\Delta \tilde{A}=\left[I-B(G B)^{-1} G\right] \Delta A=\tilde{D}_{a} F_{a}(t) E_{a} \\
\Delta \tilde{A}_{d}=\left[I-B(G B)^{-1} G\right] \Delta A_{d}=\tilde{D}_{d} F_{d}(t) E_{d}
\end{gathered}
$$

then system (1) with $w(t)=0$ in the sliding mode is asymptotically stable. Furthermore, the gain matrix $K$ is given by

$$
K=-R^{-1} B^{T} P
$$

\section{Proof:}

When the dynamics of system (1) are driven into the switching surface, we have $s(t)=0$ and $\dot{s}(t)=0$. From (4), (1) and assumption 2

$$
\dot{s}(t)=G \dot{x}(t)-G(A+B K) x(t)=0
$$

or

$$
\begin{aligned}
\dot{s}(t)=G \Delta A x(t) & +G B(I+N) u(t)+G\left(A_{d}+\Delta A_{d}\right) x(t-d) \\
& +G B_{1} w(t)-G B K x(t)=0
\end{aligned}
$$

Therefore, the equivalent control $u_{e q}(t)$ [14] is given as

$$
\begin{gathered}
u_{e q}(t)=-[G B(I+N)]^{-1}\left[G \Delta A x(t)+G\left(A_{d}+\Delta A_{d}\right) x(t-d)\right. \\
\left.+G B_{1} w(t)-G B K x(t)\right]
\end{gathered}
$$

Substituting (16) into (1), the sliding mode dynamics can be obtained

$$
\begin{gathered}
\dot{x}(t)=(A+B K) x(t)+\Delta \tilde{A} x(t) \\
+\left(\tilde{A}_{d}+\Delta \tilde{A}_{d}(t)\right) x(t-d)+\tilde{B}_{1} w(t)
\end{gathered}
$$

where $\tilde{A}_{d}, \tilde{B}_{1}, \Delta \tilde{A}$, and $\Delta \tilde{A}_{d}$ are defined in (9)-(12).

In order to examine the stability of the sliding motion described by (17), we choose the quadratic Lyapunov functional candidate as

$$
V(x, t)=x^{T}(t) P x(t)+\int_{t-d}^{t} x^{T}(\tau) R_{1} x(\tau) d \tau
$$

Observe that $V(x, t)>0, x \neq 0 ; V(x, t)=0, x=0$. Using (9)-(13), then the Lyapunov derivative along the trajectories of the sliding mode dynamics (17), when assuming $w(t)=0$, is expressed as

$$
\begin{gathered}
\dot{V}(x, t)=x^{T}(t)\left[A^{T} P+P A+R_{1}\right] x(t) \\
+2 x^{T}(t) P \tilde{D}_{a} F_{a}(t) E_{a} x(t)-2 x^{T}(t) P B R^{-1} B^{T} P x(t) \\
+2 x^{T}(t) P \tilde{A}_{d} x(t-d)+2 x^{T}(t) P \tilde{D}_{d} F(t) E_{d} x(t-d) \\
-x^{T}(t-d) R_{1} x(t-d)
\end{gathered}
$$

Applying Lemma 1 to various terms in (19) and choosing $R_{1}$ as

$$
R_{1}=\frac{1}{\varepsilon}\left(I+E_{d}^{T} E_{d}\right)
$$

Then (19) can be written as

$$
\dot{V}(x, t) \leq x^{T}(t) \Theta x(t)<0
$$

where $\Theta$ is defined in (6). The requirement of negative-definiteness of $\dot{V}(x, t)$ for stability entails that $\Theta<0$ as required by (6). If $\dot{V}(x, t)<0$ as $x \neq 0$, then $x \rightarrow 0$ as $t \rightarrow \infty$. Therefore, it is concluded that the sliding mode dynamics (17) is asymptotically stable in the sense of Lyapunov stability. The proof is completed.

\section{Theorem 2}

Consider uncertain time-delay system (1) with assumptions 1-3 and the PI switching function (4). If there exist a positive-definite symmetric solution $P \in R^{n \times n}$ such that the following inequality is satisfied

$$
\begin{gathered}
T=A^{T} P+P A-2 P B R^{-1} B^{T} P \\
+P\left(L_{1}+\gamma^{-2} \tilde{B}_{1} \tilde{B}_{1}^{T}\right) P+Q_{1}+C^{T} C<0
\end{gathered}
$$

where $R>0$, and $L_{1}$ and $Q_{1}$ is given (7) and (8), respectively. Then system (1) in the sliding mode is asymptotically stable and guarantees $\left\|T_{z w}\right\| \leq \gamma, \gamma>0$, under zero initial conditions. Furthermore, the gain matrix $K$ is given by (13).

\section{Proof:}

It can easily be seen that the inequality (22) implies the inequality (6). Therefore, it follows from Theorem 1 that system (1) with $w(t)=0$ in the sliding mode is asymptotically stable.

In the next place, to establish the upper bound $\gamma\|w(t)\|_{2}$ for the $L_{2}[0, \infty)$ norm of $z(t)$, assume the zero initial condition and let us introduce 


$$
J=\int_{0}^{\infty}\left[z^{T}(t) z(t)-\gamma^{2} w^{T}(t) w(t)\right] d t
$$

Since system (1) in the sliding mode is asymptotically stable, for any nonzero $w(t) \in L_{2}[0, \infty)$, we have

$$
\begin{gathered}
J=\int_{0}^{\infty}\left[z^{T}(t) z(t)-\gamma^{2} w^{T}(t) w(t)+\dot{V}(x, t)\right] d t \\
-x^{T}(\infty) P x(\infty)-V_{\infty}
\end{gathered}
$$

where $V_{\infty}=\lim _{t \rightarrow \infty} \int_{t-d}^{t} x^{T}(\tau) R_{1} x(\tau) d \tau \geq 0$

From (21) and $P>0$, we further have

$$
\begin{gathered}
J \leq \int_{0}^{\infty}\left[x^{T}(t)\left(\Theta+C^{T} C\right) x(t)-\gamma^{2} w^{T}(t) w(t)\right. \\
\left.+2 x^{T}(t) P \tilde{B}_{1} w(t)\right] d t
\end{gathered}
$$

Applying Lemma1,

$2 x^{T}(t) P \widetilde{B}_{1} w(t) \leq \gamma^{-2} x^{T}(t) P \widetilde{B}_{1} \tilde{B}_{1}^{T} P x(t)+\gamma^{2} w^{T}(t) w(t)$

Substituting (26) into (25), it follows from (22) that

$$
J \leq \int_{0}^{\infty} x^{T}(t) T x(t)<0
$$

This $T<0$ in (22) implies $\|z(t)\|_{2} \leq \gamma\|w(t)\|_{2}$ for any non-zero $w(t) \in L_{2}[0, \infty)$. Therefore, when $T<0$ in (22), system (1) in the sliding mode is asymptotically stable and $\left\|T_{z w}\right\| \leq \gamma$ for some prescribed $\gamma>0$. The proof is completed.

Next, Theorem 2 will be formulated by LMI approach.

\section{Theorem 3}

Consider uncertain time-delay system (1) with assumptions 1-3 and the PI switching function (4). System (1) on the switching surface is asymptotically stable and guarantees $\left\|T_{z w}\right\| \leq \gamma, \gamma>0$, under zero initial conditions if there exists a symmetric positive-definite matrix $W$ such that the following linear matrix inequality is satisfied

$$
\left[\begin{array}{cc}
\Omega & W \\
W & -Q_{2}^{-1}
\end{array}\right]<0
$$

where $\quad \Omega=W A^{T}+A W-2 B R^{-1} B^{T}+L_{1}+\gamma^{-2} \tilde{B}_{1} \tilde{B}_{1}^{T}$, $Q_{2}=Q_{1}+C^{T} C$, and the matrices $L_{1}$ and $Q_{1}$ are defined in (7) and (8), respectively. Furthermore, the gain matrix $K$ is given by

$$
K=-R^{-1} B^{T} W^{-1}
$$

Proof:

To facilitate further development, we defined

$$
W \stackrel{\Delta}{=} P^{-1}
$$

Using (29) and the Schur complement, it follows that (27) is equivalent to (22). Therefore, by Theorem 2, the existence of $W$ satisfying (27) guarantees the closed-loop system is asymptotically stable and $\left\|T_{z w}\right\| \leq \gamma$. This proof is completed.

\section{Remark 1}

A special case of system (1) occurs if the external disturbance is matched, i.e., $B_{1}=B f$, which is correspond to the case of $[6,7]$. In this case, the condition (27) in Theorem 3 reduces to

$$
\left[\begin{array}{cc}
\Omega_{1} & W \\
W & -Q_{1}^{-1}
\end{array}\right]<0
$$

where $\Omega_{1}=W A^{T}+A W-2 B R^{-1} B^{T}+L_{1}$.

\section{SLIDING MODE CONTROLLER DESIGN}

After designing the switching surface, the next step is to design an appropriate control law such that the system state trajectories are globally attracted to the switching surface in a finite time and maintain them on the surface afterwards, which is so called the reaching condition $s^{T}(t) \dot{s}(t)<0[13,14]$.

For uncertain time-delay system (1) satisfying assumptions $1-3$, the following control law is proposed:

$$
u(t)=u_{b}(t)+u_{N}(t)
$$

where $u_{b}(t)=K x(t)$ is the state feedback control, and the gain matrix $K$ is given in (28). The nonlinear switching control $u_{N}(t)$ can be expressed as

$$
u_{N}(t)=-(G B)^{-1}(\sigma+\eta(t)) \frac{s(t)}{\|s(t)\|}
$$

where $\sigma>0$ and $\eta(t)$ will be designed later. The state feedback control $u_{b}(t)$ is used to confirm the performance of the sliding mode system; the nonlinear switching control $u_{N}(t)$ is used to surmount the effect the parameter variation, state-delay and the disturbance of uncertain system (1).

\section{Theorem 4}

The uncertain time-delay system (1) with assumptions 1-3, the PI switching function (4) and the control law (31) is asymptotically stable and guarantees $\left\|T_{z w}\right\| \leq \gamma, \gamma>0$, if

$$
\begin{aligned}
\eta(t) & =\frac{M(t)}{\alpha}>0, \alpha=1-\delta_{B}>0,\|w(t)\| \leq \delta_{f} \text { and } \\
& M(t)=\sigma \delta_{B}+\delta_{B}\|G B K\|\|x(t)\|+\|G\|\left(\left\|A_{d}\right\|\|x(t-d)\|\right. \\
& \left.+\left\|D_{a}\right\|\left\|E_{a}\right\|\|x(t)\|+\left\|D_{d}\right\|\left\|E_{d}\right\|\|x(t-d)\|\right)+\left\|G B_{1}\right\| \delta_{f}
\end{aligned}
$$

\section{Proof:}

To prove the stability of uncertain system states, let us first prove that the system state trajectories can reach the sliding 
surface in a finite time and subsequently remain there, i.e., the reaching condition $s^{T}(t) \dot{s}(t)<0$ is satisfied.

From (15), we have

$$
\begin{gathered}
\dot{s}(t)=G \Delta A x(t)+G B(I+N) u(t)+G\left(A_{d}+\Delta A_{d}\right) x(t-d) \\
+G B_{1} w(t)-G B K x(t)
\end{gathered}
$$

Substituting (31)-(32) into (34)

$$
\begin{gathered}
\dot{s}(t)=-(\sigma+\eta) \frac{s(t)}{\|s(t)\|}-N(\sigma+\eta) \frac{s(t)}{\|s(t)\|}+G B N K x(t) \\
+G \Delta A x(t)+G\left(A_{d}+\Delta A_{d}\right) x(t-d)+G B_{1} w(t)
\end{gathered}
$$

Pre-multiplying (35) by $s^{T}(t)$ and using assumptions $1-3$, we obtain

$$
\begin{gathered}
s^{T}(t) \dot{s}(t)=-\sigma\|s\|-\eta\|s\|-(\sigma+\eta) N\|s\|+s^{T} G B N K x(t) \\
+s^{T} G \Delta A x(t)+s^{T} G\left(A_{d}+\Delta A_{d}\right) x(t-d)+s^{T} G B_{1} w(t) \\
\leq-\sigma\|s\|-\|s\|\left\{\eta\left(1-\delta_{B}\right)-\left[\sigma \delta_{B}+\delta_{B}\|G B K\|\|x(t)\|\right.\right. \\
+\|G\|\left(\left\|A_{d}\right\|\|x(t-d)\|+\left\|D_{a}\right\|\left\|E_{a}\right\|\|x(t)\|\right. \\
\left.\left.\left.+\left\|D_{d}\right\|\left\|E_{d}\right\|\|x(t-d)\|\right)+\left\|G B_{1}\right\|\|w(t)\|\right]\right\}
\end{gathered}
$$

Substituting (33) into (36), it yields

$$
s^{T}(t) \dot{s}(t) \leq-\sigma\|s(t)\|-\|s(t)\|\{\eta \alpha-M\}<0 \text { for }\|s(t)\| \neq 0
$$

Hence, the system state trajectories will be globally driven onto the specified switching surface $s(t)=0$ in a finite time. From Theorem 3, we know that system (1) in the sliding mode is asymptotically stable and guarantees $\left\|T_{z w}\right\| \leq \gamma, \gamma>0$, under zero initial conditions. Hence, the closed-loop system is stable and $\left\|T_{z w}\right\| \leq \gamma, \gamma>0$ is achieved. The proof is completed.

\section{Remark 2}

In order to eliminate the chattering behavior caused by $\frac{s(t)}{\|s(t)\|}$, a boundary layer is introduced around each switch surface by replacing $\frac{s(t)}{\|s(t)\|}$ in (32) by saturation function. Hence, the control law (31) can be expressed as

$$
u(t)=K x(t)-(G B)^{-1}(\sigma+\eta(t)) \operatorname{sat}\left(\frac{s(t)}{\delta}\right)
$$

The $i^{t h}$ element of $\operatorname{sat}\left(\frac{s(t)}{\delta}\right)$ is described as

$$
\operatorname{sat}\left(\frac{s_{i}(t)}{\delta_{i}}\right) \begin{cases}=\frac{s_{i}(t)}{\left|s_{i}(t)\right|} & \text { if } \quad\left|s_{i}(t)\right|>\delta_{i} \quad i=1, \cdots, m \\ =\frac{s_{i}(t)}{\delta_{i}} & \text { otherwise }\end{cases}
$$

where $\delta_{i}$ is a measure of the boundary layer thickness around the $i^{\text {th }}$ switching surface.

\section{ILLUSTRATIVE EXAMPLES}

Consider the uncertain time-delay system [6,7] described by (1) with

$$
A=\left[\begin{array}{ccc}
1 & 1 & 0 \\
0 & 1 & 0 \\
0 & 0 & -1
\end{array}\right], A_{d}=\left[\begin{array}{ccc}
0.1 & 0.1 & 0 \\
0 & 0.1 & 0 \\
0 & 0 & -0.2
\end{array}\right]
$$

$$
\begin{gathered}
D_{a}=\left[\begin{array}{ccc}
0.1 & 0.2 & 0.2 \\
0 & 0.2 & 0.2 \\
0 & 0 & 0.3
\end{array}\right], E_{a}=\left[\begin{array}{lll}
0 & 0.2 & 0.3 \\
0 & 0.3 & 0.2 \\
0 & 0.3 & 0.33
\end{array}\right] \\
F_{a}=\left[\begin{array}{ccc}
\sin (t) & 0 & 0 \\
0 & \sin (t) & 0 \\
0 & 0 & \sin (t)
\end{array}\right], d=1, D_{d}=D_{a} \\
F_{d}=\left[\begin{array}{ccc}
\cos (t) & 0 & 0 \\
0 & \cos (t) & 0 \\
0 & 0 & \cos (t)
\end{array}\right], E_{d}=E_{a}
\end{gathered}
$$

$$
\begin{gathered}
B=\left[\begin{array}{l}
0 \\
1 \\
0
\end{array}\right], \Delta B(t)=\left[\begin{array}{c}
0 \\
0.1 \cos (2 t) \\
0
\end{array}\right], B_{1}=\left[\begin{array}{c}
0 \\
0.1 \\
0
\end{array}\right] \\
w(t)=\sin (2 t), C=\left[\begin{array}{lll}
1 & 0 & 0
\end{array}\right]
\end{gathered}
$$

In this case, the external disturbance is matched. According to (4), the switching surface matrix is chosen as $G=\left[\begin{array}{lll}1 & 1 & 1\end{array}\right]$ so that $G B=1$ is nonsingular. The feedback gain matrix $K$ can be designed by using remark 1 . If we selected $\varepsilon=0.1$ and $R=1$, then the solution of (30) can be obtained as

$$
W=\left[\begin{array}{ccc}
0.0192 & -0.0437 & 0.0102 \\
-0.0437 & 0.3064 & -0.0218 \\
0.0102 & -0.0218 & 0.1364
\end{array}\right]
$$

Using (28), the gain matrix can be obtained as

$$
K=\left[\begin{array}{lll}
-10.9995 & -4.8279 & 0.0526
\end{array}\right]
$$

The control law (38) is designed as

$$
u(t)=\left[\begin{array}{lll}
-10.9995 & -4.8279 & 0.0526
\end{array}\right] x(t)
$$




$$
-(0.1+\eta(t)) \operatorname{sat}\left(\frac{s(t)}{0.1}\right)
$$

where $\eta(t)$ is given by (33).

With the initial state $x(0)=\left[\begin{array}{lll}-3 & 2 & 1\end{array}\right]^{T}$ for $t \in\left[\begin{array}{ll}-1 & 0\end{array}\right]$, the simulation results are shown in Fig. 1. Figs. 1(a), 1(b) and 1(c) show the trajectories of the states respectively. It is clearly shows that system states are successfully stabilized. Fig. 1(d) shows the control input with reasonable magnitudes. When the strategy (38) is compared with $\mathrm{Li}$ and DeCarlo's control law proposed in [6,7], it can be easily seen from Fig. 1 in [6] and Figs. 1-2 in [7] that the control exhibits disadvantageous oscillations (chattering), in the contrast, Fig1(d) in this paper shows that the control does not exhibit chattering problem. Therefore, our strategy is better than the methods proposed in $[6,7]$.

Next, the case of the system with mismatched disturbance is considered. Here, the disturbance matrix and the control output matrix can be chosen as $B_{1}=\left[\begin{array}{lll}0.1 & 0.1 & 0\end{array}\right]^{T}$ and $C=\left[\begin{array}{lll}1 & 0 & 0\end{array}\right]$, respectively. Similarly, according to (4), the switching surface matrix is chosen as $G=\left[\begin{array}{lll}1 & 1 & 1\end{array}\right]$ so that $G B=1$ is nonsingular. The feedback gain matrix $K$ can be designed by using Theorem 3. If we selected $\varepsilon=0.3, R=1$, and $\gamma=0.55$, then the solution of (27) can be obtained as

$$
W=\left[\begin{array}{ccc}
0.0161 & -0.0761 & 0.0146 \\
-0.0761 & 0.4353 & -0.0344 \\
0.0146 & -0.0344 & 0.3988
\end{array}\right]
$$

Using (28), the gain matrices can be obtained as

$$
K=\left[\begin{array}{lll}
-65.2115 & -13.5970 & 1.2192
\end{array}\right]
$$

The control law (38) is designed as

$$
\begin{aligned}
& u(t)=\left[\begin{array}{lll}
-65.2115 & -13.5970 & 1.2192
\end{array}\right] x(t) \\
& -(0.1+\eta(t)) \operatorname{sat}\left(\frac{s(t)}{0.1}\right)
\end{aligned}
$$

where $\eta(t)$ is given by (33).

With the initial state $x(0)=\left[\begin{array}{lll}-3 & 2 & 1\end{array}\right]^{T}$ for $t \in\left[\begin{array}{ll}-1 & 0\end{array}\right]$, the simulation results are shown in Fig. 2. It is clearly shows that system states are successfully stabilized and the $H_{\infty}$ performance $\left\|T_{z w}\right\| \leq 0.55$ is achieved. From these results, it is concluded that the proposed PI sliding mode controller not only stabilizes systems with state delays, mismatched parameter uncertainties and mismatched or matched external disturbances, but also yields a good performance.

\section{CONCLUSION}

In this paper, an approach has developed for the systems with state delays, mismatched parameter uncertainties and external
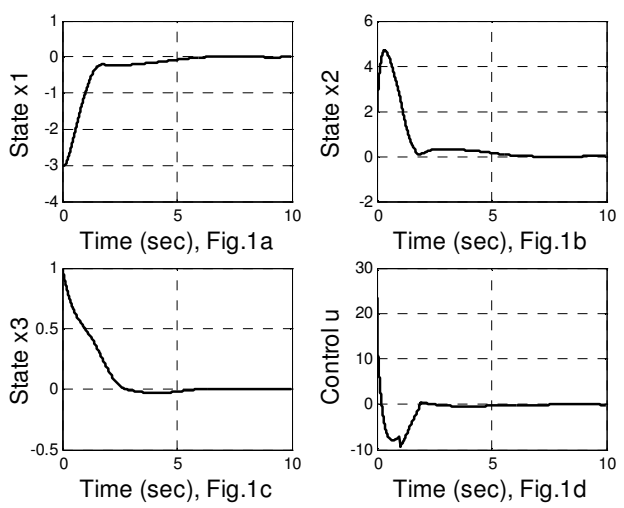

Fig. 1. (a) state $x_{1}$ (b) state $x_{2}$ (c) state $x_{3}$ (d) control input $u(t)$.
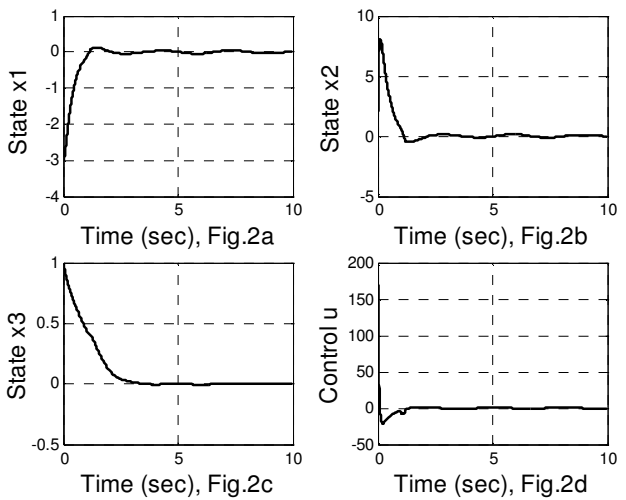

Fig. 2. (a) state $x_{1}$ (b) state $x_{2}$ (c) state $x_{3}$ (d) control input $u(t)$.

disturbance. Based on Lyapunov stability theorem and LMI- $H_{\infty}$ technique, the stability of the closed-loop system under the PI sliding mode controller is guaranteed and a prescribed $H_{\infty}$ norm bound of disturbance attenuation is achieved. Since the switching surface is designed by solving the LMIs, the control law is rather straightforward and computationally efficient. The simulation results verify the theoretical analysis and illustrate the feasibility of the proposed control methodology.

This work only considered systems with one state delay. However, it is straightforward to extend the method to systems with multiple state delays. Furthermore, it can be suitable for systems with both matched and mismatched external disturbances.

\section{REFERENCES}

1. Al-Shamali, S. A., Crisalle, O. D. and Lachman, H. A., "An Approach to Stabilize Linear Systems with State and Input Delay," Proceedings of the American Control Conference, Denver, Colorado, pp. 875-880 (2003).

2. Chou, C. H and Cheng, C. C., "Design of Adaptive Variable Structure Controller for Perturbed Time-Varying State Delay Systems," Journal of the Franklin Institute, Vol. 338, pp. 35-46 (2001).

3. Drazenovic', B., "The Invariance Condition in Variable Structure Systems," Automatica, Vol. 5, pp. 287-295 (1969).

4. Gouaisbaut, F., Dambrine, M. and Richard, J. P., "Robust Control of Delay Systems: A Sliding Mode Control Design via LMI," Systems and Control Letters, Vol. 46, pp. 219-230 (2002). 
5. Kim, J. H. and Park, H. B., “ $H_{\infty}$ State Feedback Control for Generalized Continuous / Discrete Time-Delay System,” Automatica, Vol. 35, pp. 1443-1451 (1999).

6. Li, X. and DeCarlo, R. A., "Memoryless Sliding Mode Control of Uncertain Time-Delay Systems," Proceedings of the American Control Conference, Arlingto, VA, pp. 4344-4350 (2001).

7. Li, X. and DeCarlo, R. A., "Robust Sliding Mode Control of Uncertain Time Delay Systems," International Journal of Control, , pp. 1296-1305 (2003)

8. Liu, P. L. and Su, T. J., "Robust Stability of Interval Time-Delay Systems with Delay-Dependence," Systems Control Letters, Vol. 33, pp. 231-239 (1998).

9. Petersen, I. R., “A Stabilization Algorithm for a Class of Uncertain Linear Systems", System and Control Letters, Vol. 8, pp.351-357 (1987).

10. Roh, Y. H. and Oh, J. H., "Robust Stabilization of Uncertain Input-Delay Systems by Sliding Mode Control with Delay Compensation," Automatica, Vol. 35, pp. 1861-1865 (1999).

11. Sam, Y. M., Osman, J. H. S. and Ghani, R. A., "A Class of Sliding Mode Control for Mismatched Uncertain Systems," Proceeding of Student Con- ference on Research and Development, Shah Alam, Malaysia, pp. 31-34 (2002).

12. Shyu, K. K., Tsai, Y. W. and Lai, C. K., "Sliding Mode Control for Mismatched Uncertain Systems," Electronics Letters, Vol. 34. pp. 2359-2360 (1998).

13. Slotine, J. J. and Li, W., Applied Nonlinear Control, Englewood Cliffs, NJ: Prentice-Hall, Inc. (1991).

14. Utkin, V. I., "Variable Structure Systems with Sliding Modes," IEEE Trans. Automatic Control, Vol. 22, No. 2, 212-222 (1977).

15. Xia, Y. Q., Han, J. Q. and Jia, Y. M., "A Sliding Mode Control for Linear Systems with Input and State Delays," Proceeding of the 41 st IEEE Conference on Decision and Control, Las Vega, Nevada USA, pp. 3332-3337 (2002).

16. Yu, Li, Chu, J. and Su, H., "Robust Memoryless $H_{\infty}$ Controller Design for Linear Time-delay Systems with Norm-bounded Time-varying Uncertainty," Automatica, Vol. 32, No. 12, pp. 1759-1762 (1996).

17. Zribi, M. and Mahmoud, M. S., " $H_{\infty}$ Control Design for System with Multiple Delays," Computer and Electrical Engineering, Vol. 25, pp. 451-475 (1999). 